\section{A) Check for updates}

Cite this: Org. Chem. Front., 2021, 8 4138

Received 26th April 2021,

Accepted 6th May 2021

DOI: $10.1039 /$ d1qo00644d

rsc.li/frontiers-organic

\title{
Exploiting coordination geometry to tune the dimensions and processability of metallosupramolecular polymers $\uparrow$
}

\author{
Nils Bäumer, ${ }^{a}$ Kalathil K. Kartha, (D) a Stefan Buss, (D) b,c Jasnamol P. Palakkal, (D) d \\ Cristian A. Strassert (iD b,c and Gustavo Fernández (iD *a
}

\begin{abstract}
Achieving precise control over the morphology, dimensions and processability of functional materials is a key but challenging requirement for the fabrication of smart devices. To address this issue, we herein compare the self-assembly behavior of two new Pt(॥) complexes that differ in the molecular and coordination geometry through implementation of either a monodentate (pyridine) or bidentate (bipyridine) ligand. The molecular preorganization of the bipyridine-based complex enables effective self-assembly in solution involving Pt...Pt interactions, while preserving aggregate solubility. On the other hand, increased steric effects of the linear bispyridine-based complex hinder an effective preorganization leading to poorly solvated aggregates when a critical concentration is exceeded.
\end{abstract}

\section{Introduction}

The desire to develop functional materials has helped kickstart numerous approaches to tune supramolecular self-assembly in solution. ${ }^{1-5}$ Particularly, to achieve precise properties, a constant balance between tailored aggregation, nanoscale morphology and processability has to be maintained. ${ }^{6,7}$ In this regard, living supramolecular polymerization represents a milestone towards controlling nanoscale morphology and supramolecular size distributions with defined aspect ratios. ${ }^{8-13}$ Similarly, secondary nucleation events at the outer sphere of a primary supramolecular assembly can be exploited to obtain a stepwise increase in size, or to acquire otherwise inaccessible morphologies. ${ }^{14,15}$ However, achieving similar dimensional control based on molecular design remains a challenging task towards application.

\footnotetext{
${ }^{a}$ Organisch Chemisches Institut, Universität Münster, Corrensstraße 36, 48149 Münster, Germany. E-mail: fernandg@uni-muenster.de

${ }^{b}$ Institut für Anorganische und Analytische Chemie, CiMIC, SoN, Westfälische Wilhelms-Universität Münster, Corrensstraße 28/30, 48149 Münster, Germany ${ }^{c}$ CeNTech, Westfälische Wilhelms-Universität Münster, Heisenbergstraße 11, 48149 Münster, Germany

${ }^{d}$ Institute of Materials Science, Technische Universität Darmstadt, 64287 Darmstadt, Germany

$\dagger$ Electronic supplementary information (ESI) available: Experimental section, synthetic schemes; complete spectroscopic characterization; variable temperature/solvent UV-Vis studies; ${ }^{1} \mathrm{H}$ NMR studies; photoluminescence studies; AFM imaging, phosphorescence lifetime measurements, XRD diffraction. See DOI: 10.1039/d1qo00644d
}

Dynamic supramolecular polymers with properties such as conductivity, self-healing or desired photophysical behaviour are prime candidates for the design of smart devices, ${ }^{16,17}$ as the bulk property is greater than in the monomeric state or self-assembly is a prerequisite for the emergence of certain properties. ${ }^{18,19}$ Nevertheless, some major challenges still hamper the progress of supramolecular polymers towards application, particularly in terms of finding the right balance between aggregation and solubility, ${ }^{13,20,21}$ which largely controls processability. ${ }^{22,23}$ Accordingly, design strategies that can attenuate initial steric hindrance and allow for a more controllable self-assembly process while preserving solubility and desired functional properties are a central goal in supramolecular chemistry. ${ }^{24,25}$

Recently, we have demonstrated that the coordination geometry serves to control molecular preorganization events that, in turn, regulate the packing modes, energy landscapes and luminescence of metallosupramolecular polymers (Scheme S3†). ${ }^{26}$ However, nanoscale morphology and aggregation mechanism were influenced in a minor way. To further elucidate the influence of coordination and molecular geometry on self-assembly, we have modified our previous design to obtain complex 1 (Scheme 1). This chemical modification is expected to decrease the aggregation propensity and drive the system towards different pathways and molecular arrangements (see $\mathrm{ESI} \uparrow$ for expanded discussion). Further, a linear bispyridinebased complex 2 serves as reference compound, as the molecular composition remains identical besides the addition of formally two hydrogen atoms (Scheme 1). Based on our previous work, we hypothesize that the molecular preorganization exhibi- 


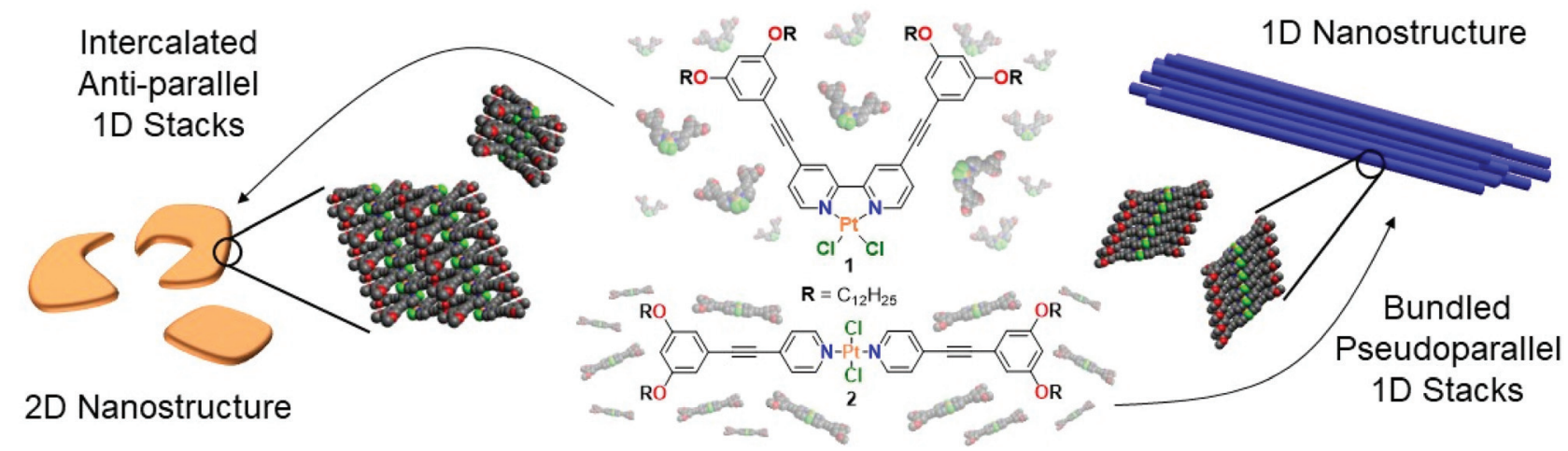

Scheme 1 Molecular structures of 1 and 2 and schematic representation of their self-assembly in $\mathrm{MCH}$.

ted by the V-shaped complex 1 should lead to a preferential selfassembly and improved solvation in comparison to 2 . This linear complex is expected to undergo a less controllable selfassembly into large superstructures due to the steric hindrance induced by the out-of-plane orientation of the chlorido ligands.

\section{Results and discussion}

To examine the aggregation tendency of $\mathbf{1}$, we initially used solvent-dependent UV/Vis studies at low concentration $(c=10$ $\times 10^{-6} \mathrm{M}$, Fig. S9 $\dagger$ ). In accordance with previous reports on similar complexes, we identified low polarity solvents such as methylcyclohexane ( $\mathrm{MCH})$ as suitable candidates for aggregation studies. Subsequently, we turned to variable temperature (VT)-UV/Vis in $\mathrm{MCH}\left(c=3 \times 10^{-6} \mathrm{M}\right)$ in order to get a better understanding of the self-assembly mechanism and preliminary information on the supramolecular packing mode (Fig. 1). 1 shows two lower energy absorption bands centered
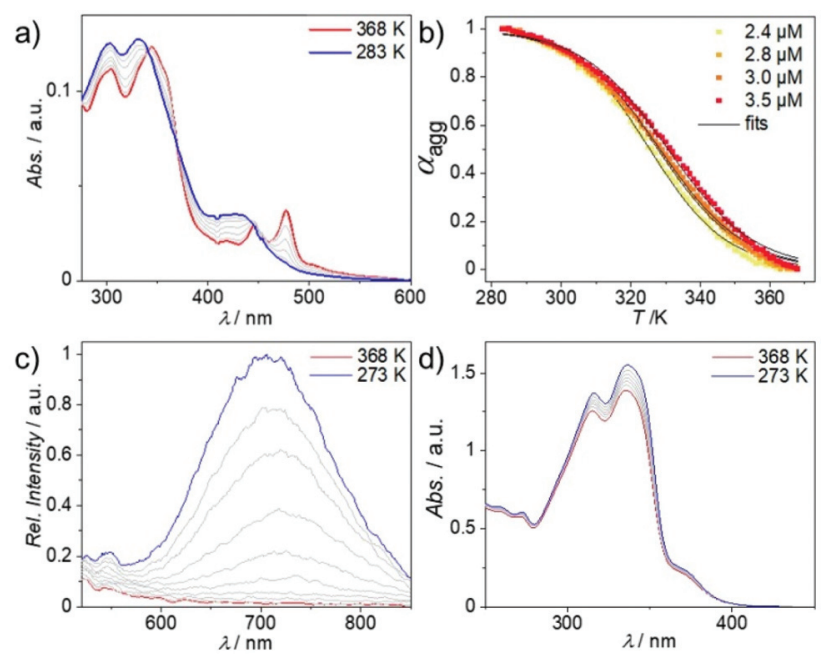

Fig. 1 VT-UV/Vis (a) and VT-photoluminescence (c) of 1 at $c=3 \times 10^{-6}$ $\mathrm{M}$ in $\mathrm{MCH}$. (b) $\alpha_{\text {agg }}$ plotted against $T$ fitted to the isodesmic model. ${ }^{29}$ (d) VT-UV/Vis of 2 at $3 \times 10^{-5} \mathrm{M}$ in $\mathrm{MCH}$. at 445 and $475 \mathrm{~nm}$ at high temperature (368 K), which can be attributed to a transition into a metal-to-ligand charge transfer state (MLCT, see Fig. S10† and subsequent discussion) of the molecularly dissolved state. ${ }^{27}$ Cooling to $283 \mathrm{~K}$ leads to the depletion of the absorption at $475 \mathrm{~nm}$ and a concurrent increase of a broader absorption between 400 and $450 \mathrm{~nm}$ with a maximum at $430 \mathrm{~nm}$ (Fig. 1a), pointing to the formation of $H$-type aggregates. ${ }^{28}$ Both high energy absorption bands show a similar hypsochromic shift of 5 and $15 \mathrm{~nm}$, respectively. Plotting the degree of aggregation $\left(\alpha_{\text {agg }}\right)$ calculated at $\lambda=$ $475 \mathrm{~nm} v s$. the temperature $(T)$ shows a clear sigmoidal transition between the monomer and the aggregated state of $\mathbf{1}$ (an identical trend is observed in denaturation studies, see Fig. S11†). Consequently, the aggregation of 1 can be best described by the equal $K$ (isodesmic) model ${ }^{29}$ (Fig. 1b, for detailed thermodynamic analysis see ESI; Fig. S12 $\dagger$ ) and the obtained fits accurately replicate the spectral data at various concentrations. These findings are in sharp contrast to the cooperative mechanism observed for a structurally related bipyridine-based Pt(II) complex with a larger $\pi$-system. ${ }^{26}$ Thus, the reduction of the $\pi$-core leads to a switch from a cooperative to an isodesmic aggregation mechanism, which is also reflected in the absence of pathway complexity for $\mathbf{1}$. These results point to a significant change in the intermolecular interactions governing the self-assembly process of both derivatives (vide infra). Additionally, we performed VT-photoluminescence studies of $\mathbf{1}$ using identical concentration, solvent and temperature range (Fig. 1c). During cooling from $368 \mathrm{~K}$ to $273 \mathrm{~K}$, a broad emission between 600 and $850 \mathrm{~nm}$ progressively arises. Based on previous reports, we propose that this emission stems from the emergence of ${ }^{3}$ MMLCT states in combination with the decrease in non-radiative decay pathways caused by the temperature drop. ${ }^{30}$ Interestingly, the molecularly dissolved state does not show emissive behavior in the low energy region in a variety of good solvents, indicating that an aggregation-induced ${ }^{3}$ MMLCT excited state is observed. This is also supported by a more detailed solvent-dependent study (Fig. S14 and $15 \dagger$ ). Subsequently, phosphorescence lifetime measurements of the aggregated form of 1 were performed after cooling a hot monomeric solution $\left(c=10 \times 10^{-6}\right.$ 
$\mathrm{M}, T=368 \mathrm{~K})$ to ambient conditions. Agg1 shows an average lifetime of 116 ns (amplitude weighted) under degassed conditions, while further cooling to $77 \mathrm{~K}$ extends the long average lifetimes to roughly $14 \mu \mathrm{s}$ (Fig. S16-S20†). These lifetimes in combination with the low energy featureless emission profile support our assignment to ${ }^{3}$ MMLCT states.

Comparing molecularly preorganized 1 with the linear compound 2 clearly shows the drastic influence of changing the coordination and molecular geometry. Solvent-dependent UV/ Vis spectra of 2 disclose solvatochromic effects on the absorption profile. 2 exhibits only one broad absorption band at $330 \mathrm{~nm}$ in moderately polar solvents (chloroform and DCM; Fig. S21†), whereas decreasing the solvent polarity to $n$-hexane or $\mathrm{MCH}$ leads to a splitting of the broad absorption band into two bands at 315 and $335 \mathrm{~nm}$ (Fig. S22, for further details, see the ESI $\dagger$ ). Concomitantly, a shoulder at $375 \mathrm{~nm}$ rises upon polarity decrease. VT-UV/Vis and VT photoluminescence studies reveal no signs of aggregation apart from a minor increase in absorption or emission (Fig. 1d, S23 and 24†), which can be assigned to the planarization of the aromatic system in the molecularly dissolved state upon cooling. ${ }^{31}$ This hypothesis is supported by the observation of well-defined sharp signals and only minor shifts in solvent-dependent ${ }^{1} \mathrm{H}$ NMR spectra even at high $\mathrm{MCH}-\mathrm{d}_{14}$ contents in $\mathrm{CDCl}_{3}$ (Fig. S25 $\dagger$ ), which is reaffirmed by $\mathrm{VT}^{-}{ }^{1} \mathrm{H}$ NMR in low polarity solvents (Fig. S26 and 27†). These results again highlight the significance of coordination geometry, as the linear compound 2 cannot effectively self-assemble in contrast to a previously reported compound with a larger aromatic surface, ${ }^{26}$ whereas 1 engages in a new aggregation pathway.

Subsequently, we employed solvent- as well as temperaturedependent ${ }^{1} \mathrm{H}$ NMR spectroscopy in order to understand the influence of coordination geometry and molecular preorganization on the packing mode of $\mathbf{1}$. The stepwise increase of the volume fraction of $\mathrm{MCH}-\mathrm{d}_{14}$ in $\mathrm{CDCl}_{3}$ leads to the aggregation of 1 at ambient conditions, which was also confirmed by solvent-dependent UV/Vis (Fig. 2a). The signals corresponding to the alpha proton $\mathrm{H}_{\mathrm{a}}$ of the bipyridine exhibit the most significant upfield shift from 9.82 to $9.48 \mathrm{ppm}$, indicating their involvement in aromatic interactions. ${ }^{32,33}$ Further, the signal corresponding to the adjacent proton $\mathrm{H}_{\mathrm{b}}$ exhibits only a very minor upfield shift (7.60 to $7.49 \mathrm{ppm}$ ), indicating weaker intermolecular interactions. The remaining signal corresponding to the bipyridine moiety $\left(\mathrm{H}_{\mathrm{c}}\right)$, however, shows a different behavior. Initially, up to $60 \% \mathrm{MCH}^{-\mathrm{d}_{14}}$ in $\mathrm{CDCl}_{3}$, a downfield shift can be observed indicating the proximity of an electron rich group (such as $\mathrm{O}$ or $\mathrm{Cl}$ ). ${ }^{34}$ At $\mathrm{MCH}$ fractions above $60 \%$, a second process sets in, namely a minor upfield shift accompanied with significant broadening. The two proton signals corresponding to the peripheral phenyl rings exhibit a similar behavior, namely a downfield shift at high chloroform contents, followed by signal broadening upon increasing the

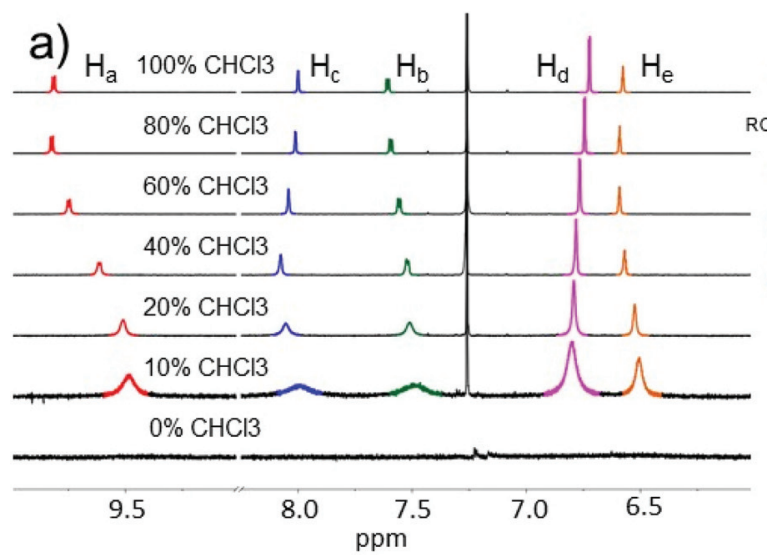

C)

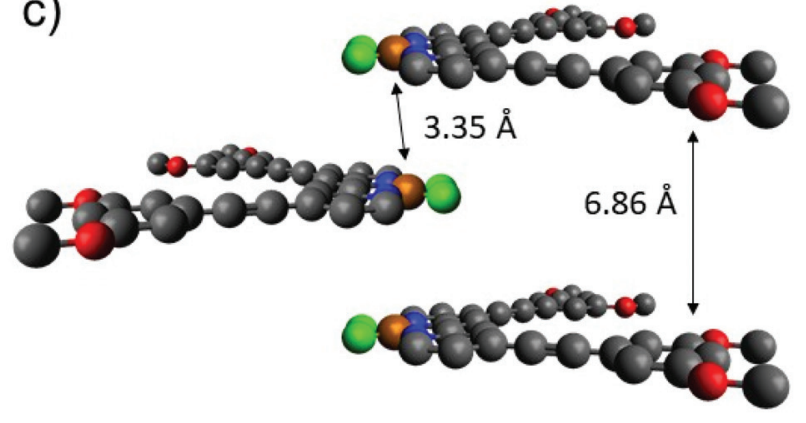

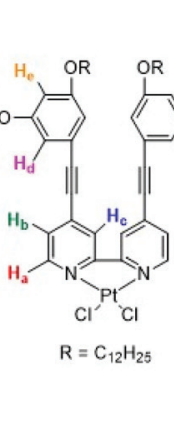
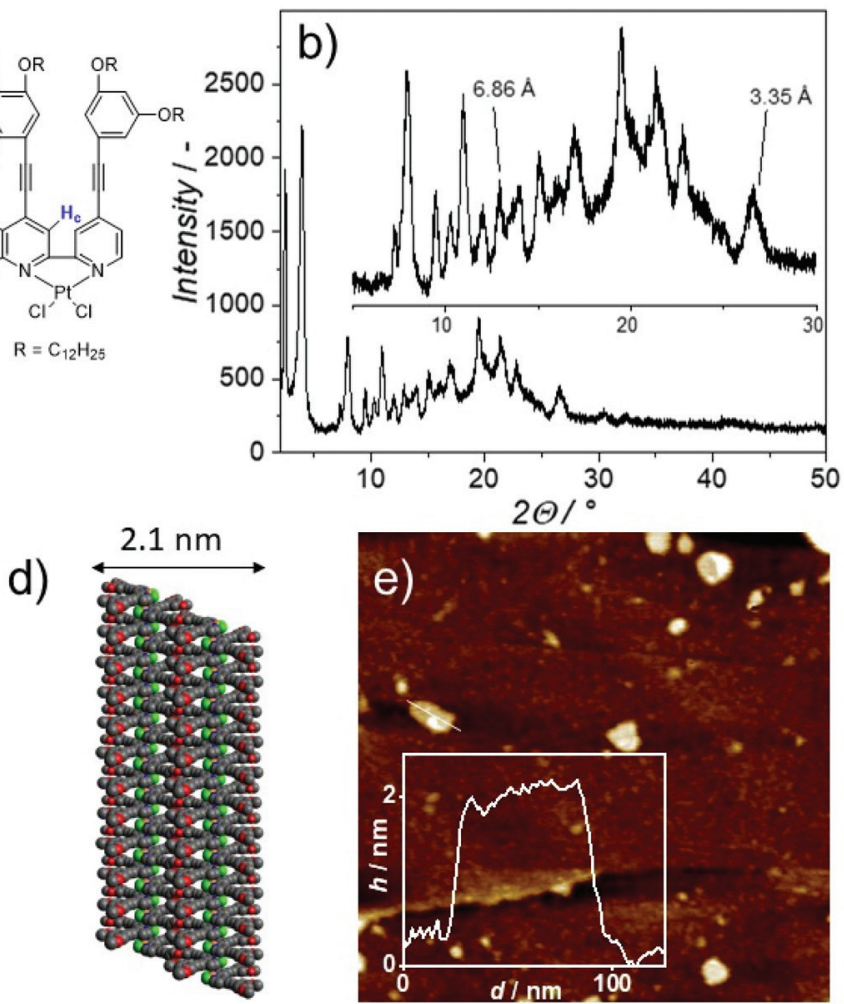

Fig. 2 (a) Partial ${ }^{1} \mathrm{H}$ NMR spectra of 1 in $\mathrm{MCH}-\mathrm{d}_{14} / \mathrm{CDCl}_{3}$ mixtures $\left(c=1 \times 10^{-3} \mathrm{M}, T=298 \mathrm{~K}\right.$ ) along with proton assignment. (b) PXRD diffraction pattern of Agg1, sample prepared from a solution in $\mathrm{CHCl} / \mathrm{MCH}\left(2: 8, c=7.5 \times 10^{-3} \mathrm{M}\right)$. (c and d) Schematic representation of the proposed packing mode of 1 in one (c) and two dimensions (d). (e) AFM height image of Agg1, inset: height profile. 
$\mathrm{MCH}$ content. Moreover, it can be observed that the signals corresponding to the bipyridine moiety show much greater signal broadening compared to the signals of the phenyl ring. VT ${ }^{1} \mathrm{H}$ NMR at intermediate solvent mixtures confirms this behavior (Fig. S28 and 29†). Moreover, ROESY NMR (see ESI for detailed discussion, Fig. S30†) suggests close intermolecular contacts between the alkyl chains and the aromatic protons of the neighboring bipyridine moiety. In combination with the intermolecular close contact between the $\mathrm{OCH}_{2}$ methylene unit and the alpha proton of the central bipyridine, we infer an antiparallel arrangement of the molecules within a 1D stack with possible interdigitation yielding 2D architectures. This proposed packing mode was further investigated by powder X-ray diffraction (PXRD). Numerous sharp reflexes can be observed correlating to distances between 4.5 and $10 \AA$. In particular a single reflex at $2 \Theta=26.5^{\circ}$ corresponding to a distance of $3.35 \AA$ can be observed (Fig. 2b). In accordance with the observed lifetimes, we attribute this reflex to close Pt...Pt contacts, which lead to ${ }^{3}$ MMLCT excited states. However, no second reflex within a similar range can be observed, which suggests that the molecules do not stack in a parallel fashion, otherwise the $\pi-\pi$ distance should also be observable. ${ }^{35}$ Hence, the distance between the aromatic units should be among the reflexes between 10 and $5 \AA$. In fact, the observation of a reflex at $6.86 \AA$, which we tentatively assign to the distance between aromatic moieties, supports this hypothesis. The proposed antiparallel arrangement further offers a plethora of diagonal spacings, which could explain the remaining reflexes. Combining the results from NMR and PXRD, we propose a model depicting the intermolecular packing (Fig. 2c and Fig. S31, 32 $\dagger$ ). The monomers of 1 stack in an antiparallel fashion, with the bipyridine moieties constituting the inner core of the 1D stacks leading to close intermolecular Pt...Pt contacts (Fig. 2d), in good agreement with the observed ${ }^{3}$ MMLCT emission (vide supra). The alkyl chains point towards the outside of the stack, effectively shielding the aromatic core from the nonpolar solvent. Additionally, this model offers a reasonable explanation for the more pronounced signal broadening of the bipyridine signals $\left(\mathrm{H}_{\mathrm{a}, \mathrm{b}, \mathrm{c}}\right)$ compared to the phenyl signals $\left(\mathrm{H}_{\mathrm{d}, \mathrm{e}}\right)$ observed in solvent-dependent $\mathrm{NMR}$, as the central part of the stack is more significantly involved in intermolecular interactions. This effective rearrangement in comparison to our previously reported compound ${ }^{26}$ clearly reflects that different coordination geometries can allow molecular entities to engage in new aggregation pathways balancing attractive intermolecular interaction and steric demand, which may not be the case for all ligand systems (vide infra).

In order to correlate the packing motif with the nanoscale morphology, we turned to atomic force microscopy (Fig. 2e and Fig. S33-35†). The aggregates of 1 can be best described as small 2D plates with a homogenous height of $2.1 \mathrm{~nm}$, which matches well with the alignment of multiple 1D stacks. Additionally, keeping a highly concentrated solution of $\mathbf{1}$ in nonpolar solvents over time leads to the precipitation of microcrystalline assemblies consisting of smaller $2 \mathrm{D}$ plates (Fig. S36-38†). We speculate that this hierarchical level in the

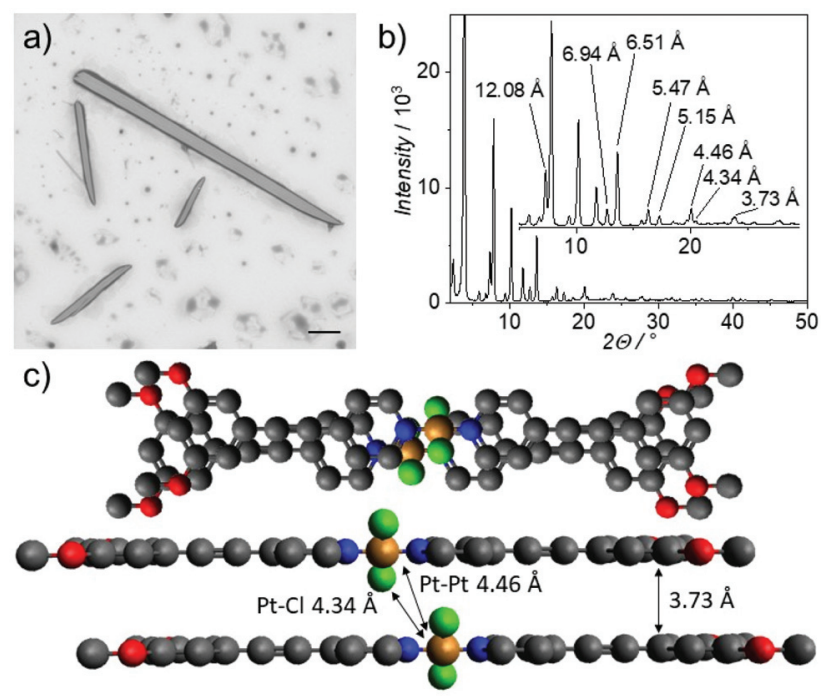

Fig. 3 (a) SEM image of Agg2, the scale bar corresponds to $10 \mu \mathrm{m}$. (b) PXRD diffraction pattern of Agg2. Sample prepared from a solution in $\mathrm{MCH}\left(c=1 \times 10^{-3} \mathrm{M}\right)$. (c) Representation of the proposed packing mode of Agg2.

self-assembly behavior is the result of further stack intercalation leading to a 3D growth.

Similarly, keeping highly concentrated solutions of 2 (c $>1 \mathrm{mM}$ ) in solvents of low polarity ( $\mathrm{MCH}$ content $>80 \%$, Fig. S36†) also leads to the formation of large needles with lengths between 10 and 200 microns and widths between 1 and 10 microns, as visualized by scanning electron microscopy (SEM, Fig. 3a and S39†). Upon closer inspection, it becomes apparent that these large structures consist of smaller fibers, which bundle together yielding larger morphologies (Fig. S40†). The microcrystalline nature of the needle-like structures is reflected in the well-defined diffraction pattern observed in PXRD (Fig. 3b). Comparing this pattern with molecular structures in the crystal of structurally related bispyridyldichlorido $\mathrm{Pt}(\mathrm{II})$ complexes, ${ }^{36}$ we derive an almost parallel molecular packing with a small translational displacement to alleviate steric constraints (see Fig. S41† and subsequent discussion). These results highlight that modifying the coordination geometry to achieve molecular preorganization and alleviate steric constrains can enable spatiotemporal control over self-assembly and precipitation effects.

\section{Conclusions}

In conclusion, we have analysed the role of coordination and molecular geometry in controlling the dimensionality and processability of self-assembled structures. To this end, we have compared the self-assembly behaviour of two Pt(II) complexes with a different degree of preorganization (molecularly preorganized bipyridine-based $\mathbf{1}$ and non-preorganized bispyridine-based 2) in nonpolar media. 1 self-assembles via the isodesmic mechanism into antiparallel 1D stacks that are stabil- 
ized by metal-metal interactions. Lateral interchain interactions between the stacks induce a hierarchical growth into solvated 2D plates and, subsequently to $2 \mathrm{D}$ microcrystalline plates. In sharp contrast, non-preorganized 2 does not selfassemble into solvated aggregates in solution. Instead, increasing the concentration in nonpolar media induces a sudden self-assembly process that leads to rapid precipitation of large bundles of fibers. Our results disclose coordination geometry as an effective tool to achieve dimensional control and processability, which is a prerequisite for the design and application of functional nanomaterials.

\section{Author contributions}

The manuscript was written through contribution of all authors. All authors have given approval to the final version of the manuscript.

\section{Conflicts of interest}

There are no conflicts to declare.

\section{Acknowledgements}

N. B., K. K. K. and G. F. acknowledge the European Commission (ERC-StG-2016 SUPRACOP-715923) for funding. C. A. S. acknowledges the DFG (EXC 1003 Cluster of Excellence "Cells in Motion"; SPP "Light Controlled Reactivity of Metal Complexes") for funding. J. P. P. acknowledges the DFG 429646908 for funding. We thank Prof. Lambert Alff for the access to the PXRD measurements.

\section{Notes and references}

1 L. Voorhaar and R. Hoogenboom, Supramolecular polymer networks: hydrogels and bulk materials, Chem. Soc. Rev., 2016, 45, 4013-4031.

2 F. Seidi, A. A. Shamsabadi, M. Amini, M. Shabanian and D. Crespy, Functional materials generated by allying cyclodextrin-based supramolecular chemistry with living polymerization, Polym. Chem., 2019, 10, 3674-3711.

3 O. J. G. M. Goor, S. I. S. Hendrikse, P. Y. W. Dankers and E. W. Meijer, From supramolecular polymers to multi-component biomaterials, Chem. Soc. Rev., 2017, 46, 6621-6637.

4 T. Aida, E. W. Meijer and S. I. Stupp, Functional supramolecular polymers, Science, 2012, 335, 813-817.

5 S. S. Babu, V. K. Praveen and A. Ajayaghosh, Functional $\pi$-gelators and their applications, Chem. Rev., 2014, 114, 1973-2129.

6 S. J. Ananthakrishnan, E. Varathan, E. Ravindran, N. Somanathan, V. Subramanian, A. B. Mandal, J. D. Sudha and R. A. Ramakrishnan, Solution processable fluorene- fluorenone oligomer with aggregation induced emission enhancement, Chem. Commun., 2013, 49, 10742-10744.

7 C. Li, R. S. Nobuyasu, Y. Wang, F. B. Dias, Z. Ren, M. R. Bryce and S. Yan, Solution-Processable Thermally Activated Delayed Fluorescence White OLEDs Based on Dual-Emission Polymers with Tunable Emission Colors and Aggregation-Enhanced Emission Properties, Adv. Opt. Mater., 2017, 5, 1700435.

8 S. Ogi, K. Sugiyasu, S. Manna, S. Samitsu and M. Takeuchi, Living supramolecular polymerization realized through a biomimetic approach, Nat. Chem., 2014, 6, 188-195.

9 K. Zhang, M. C.-L. Yeung, S. Y.-L. Leung and V. W.-W. Yam, Living supramolecular polymerization achieved by collaborative assembly of platinum(II) complexes and block copolymers, Proc. Natl. Acad. Sci. U. S. A., 2017, 114, 1184411849.

10 Z. M. Hudson, D. J. Lunn, M. A. Winnik and I. Manners, Colour-tunable fluorescent multiblock micelles, Nat. Commun., 2014, 5, 3372.

11 T. Fukui, S. Kawai, S. Fujinuma, Y. Matsushita, T. Yasuda, T. Sakurai, S. Seki, M. Takeuchi and K. Sugiyasu, Control over differentiation of a metastable supramolecular assembly in one and two dimensions, Nat. Chem., 2017, 9, 493499.

12 W. Wagner, M. Wehner, V. Stepanenko, S. Ogi and F. Würthner, Living Supramolecular Polymerization of a Perylene Bisimide Dye into Fluorescent J-Aggregates, Angew. Chem., Int. Ed., 2017, 56, 16008-16012.

13 N. Sasaki, J. Yuan, T. Fukui, M. Takeuchi and K. Sugiyasu, Control over the Aspect Ratio of Supramolecular Nanosheets by Molecular Design, Chem. - Eur. J., 2020, 26, 7840-7846.

14 N. Sasaki, M. F. J. Mabesoone, J. Kikkawa, T. Fukui, N. Shioya, T. Shimoaka, T. Hasegawa, H. Takagi, R. Haruki, N. Shimizu, et al., Supramolecular double-stranded Archimedean spirals and concentric toroids, Nat. Commun., 2020, 11, 3578.

15 S. Datta, Y. Kato, S. Higashiharaguchi, K. Aratsu, A. Isobe, T. Saito, D. D. Prabhu, Y. Kitamoto, M. J. Hollamby, A. J. Smith, et al., Self-assembled poly-catenanes from supramolecular toroidal building blocks, Nature, 2020, 583, 400-405.

16 Y. Ai, Y. Li, H. L.-K. Fu, A. K.-W. Chan and V. W.-W. Yam, Aggregation and Tunable Color Emission Behaviors of l-Glutamine-Derived Platinum(II) Bipyridine Complexes by Hydrogen-Bonding, $\pi-\pi$ Stacking and Metal-Metal Interactions, Chem. - Eur. J., 2019, 25, 5251-5258.

17 S. Carrara, A. Aliprandi, C. F. Hogan and L. De Cola, Aggregation-Induced Electrochemiluminescence of Platinum(II) Complexes, J. Am. Chem. Soc., 2017, 139, 14605-14610.

18 A. S.-Y. Law, L. C.-C. Lee, M. C.-L. Yeung, K. K.-W. Lo and V. W.-W. Yam, Amyloid Protein-Induced Supramolecular SelfAssembly of Water-Soluble Platinum(II) Complexes: A Luminescence Assay for Amyloid Fibrillation Detection and 
Inhibitor Screening, J. Am. Chem. Soc., 2019, 141, 1857018577.

19 S. Chakraborty, S. Varghese and S. Ghosh, Supramolecular Nanowires from an Acceptor-Donor-Acceptor Conjugated Chromophore, Chem. - Eur. J., 2019, 25, 16725-16731.

20 P. K. Hashim, J. Bergueiro, E. W. Meijer and T. Aida, Supramolecular Polymerization: A Conceptual Expansion for Innovative Materials, Prog. Polym. Sci., 2020, 105, 101250.

21 J. Matern, K. K. Kartha, L. Sánchez and G. Fernández, Consequences of hidden kinetic pathways on supramolecular polymerization, Chem. Sci., 2020, 11, 6780-6788.

22 Y.-H. Cheng, H.-L. Wong, E. Y.-H. Hong, M.-Y. Leung, S.-L. Lai and V. W.-W. Yam, Design and Synthesis of Solution-Processable Donor-Acceptor Dithienophosphole Oxide Derivatives for Multilevel Organic Resistive Memories, ACS Mater. Lett., 2020, 2, 1590-1597.

23 W.-L. Cheung, S.-L. Lai, W.-K. Kwok, M.-C. Tang, C.-H. Lee, M.-Y. Chan and V. W.-W. Yam, Solution-processable cyclometalated gold(III) complexes for high-brightness phosphorescent white organic light-emitting devices, J. Mater. Sci., 2020, 55, 9686-9694.

24 S. Xue, Y. Wu, Y. Lu, X. Xu, Q. Sun and W. Yang, A pair of conjoined donor-acceptor butterflies as promising solution-processable aggregation-enhanced emission FR/NIR EL emitters, J. Mater. Chem. C, 2017, 5, 11700-11707.

25 D. Samanta, A. Singh, P. Verma, S. Bhattacharyya, S. Roy and T. K. Maji, Photoswitchable J-Aggregated Processable Organogel by Integrating a Photochromic Acceptor, J. Org. Chem., 2019, 84, 10946-10952.

26 N. Bäumer, K. K. Kartha, S. Buss, I. Maisuls, J. P. Palakkal, C. A. Strassert and G. Fernández, Tuning Energy Landscapes and Metal-Metal Interactions in Supramolecular Polymers regulated by Coordination Geometry, Chem. Sci., 2021, 12, 5236.

27 P. M. Gidney, R. D. Gillard and B. T. Heaton, Solvent effects on the electronic spectra of some 2,2'-bipyridyl palladium (II) and platinum(II) complexes, J. Chem. Soc., Dalton Trans., 1973, 132-134.
28 M. Kasha, H. R. Rawls and M. A. El-Bayoumi, The Exciton Model in Molecular Spectroscopy, Pure Appl. Chem., 1965, 11, 371-392.

29 M. M. J. Smulders, M. M. L. Nieuwenhuizen, T. F. A. de Greef, P. van der Schoot, A. P. H. J. Schenning and E. W. Meijer, How to distinguish isodesmic from cooperative supramolecular polymerisation, Chem. - Eur. J., 2010, 16, 362-367.

30 V. M. Miskowski, V. H. Houlding, C.-M. Che and Y. Wang, Electronic spectra and photophysics of platinum(II) complexes with alpha.-diimine ligands. Mixed complexes with halide ligands, Inorg. Chem., 1993, 32, 2518-2524.

31 D. S. Philips, K. K. Kartha, A. T. Politi, T. Krüger, R. Q. Albuquerque and G. Fernández, Interplay between H-Bonding and Preorganization in the Evolution of SelfAssembled Systems, Angew. Chem., Int. Ed., 2019, 58, 47324736.

32 C. Giessner-Prettre, B. Pullman, P. N. Borer, L. S. Kan and P. O. P. Ts'o, Ring-current effects in the nmr of nucleic acids: A graphical approach, Biopolymers, 1976, 15, 22772286.

33 S. Lahiri, J. L. Thompson and J. S. Moore, Solvophobically Driven $\pi$-Stacking of Phenylene Ethynylene Macrocycles and Oligomers, J. Am. Chem. Soc., 2000, 122, 1131511319.

34 X. Lu, Z. Guo, C. Sun, H. Tian and W. Zhu, Helical assembly induced by hydrogen bonding from chiral carboxylic acids based on perylene bisimides, J. Phys. Chem. B, 2011, 115, 10871-10876.

35 L. Herkert, P. Selter, C. G. Daniliuc, N. Bäumer, J. P. Palakkal, G. Fernández and M. R. Hansen, Tuning the Molecular Packing of Self-Assembled Amphiphilic $\mathrm{Pt}^{\mathrm{II}}$ Complexes by Varying the Hydrophilic Side-Chain Length, Chem. - Eur. J., 2021, 27, 4617-4626.

36 N. K. Allampally, M. J. Mayoral, S. Chansai, M. C. Lagunas, C. Hardacre, V. Stepanenko, R. Q. Albuquerque and G. Fernández, Control over the Self-Assembly Modes of Pt (II) Complexes by Alkyl Chain Variation: From Slipped to Parallel $\pi$-Stacks, Chem. - Eur. J., 2016, 22, 7810-7781. 